\title{
ANALYSIS OF THE POLYCRYSTALLINE MICROSTRUCTURE OF AIMgSc ALLOY OBSERVED BY 3D EBSD
}

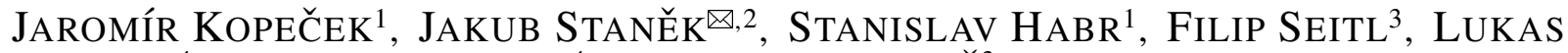 \\ PETRICH $^{4}$, VOLKER SCHMIDT ${ }^{4}$ AND VIKTOR BENEŠ ${ }^{3}$ \\ ${ }^{1}$ ZSU - Institute of Physics of the CAS, Department of Functional Materials, Na Slovance 2, 18200 Prague 8, \\ Czech Republic, ${ }^{2}$ Department of Mathematics Education, Faculty of Mathematics and Physics, Charles \\ University, 18675 Prague, Czech Republic, ${ }^{3}$ Department of Probability and Mathematical Statistics, Faculty of \\ Mathematics and Physics, Charles University, 18675 Prague, Czech Republic, ${ }^{4}$ Institute of Stochastics, Faculty \\ of Mathematics and Economics, Ulm University, 89069 Ulm, Germany \\ e-mail: kopecek@fzu.cz, stanekj@karlin.mff.cuni.cz, seitlf@seznam.cz, lukas.petrich@uni-ulm.de, \\ benesv@karlin.mff.cuni.cz, volker.schmidt@uni-ulm.de \\ (Received August 21, 2019; revised November 29, 2019; accepted December 2, 2019)
}

\begin{abstract}
The aim of this paper is to evaluate an ambitious imaging experiment and to contribute to the methodology of statistical inference of the three-dimensional microstructure of polycrystalline materials. The microstructure of the considered $\mathrm{Al}-3 \mathrm{Mg}-0.2 \mathrm{Sc}$ alloy was investigated by three-dimensional electron backscattered diffraction (3D-EBSD), i.e., tomographic imaging with xenon plasma focused ion beam (Xe-FIB) alongside EBSD. The samples were subjected to severe plastic deformations by equal channel angular pressing (ECAP) and annealed subsequently prior to the mapping. First we compared the misorientation level needed for a reliable segmentation of grains distinguishing between conventional evaluation of two-dimensional cuts and the 3D data set. Then, using methods of descriptive spatial statistics, various morphological characteristics of a large number of grains were analyzed, as well as the crystallographic texture and the spatial distribution of grain boundaries. According to the results stated so far in the literature, an even microstructure was expected, nevertheless local inhomogeneities in grains and grain boundaries with regard to their size, texture and spatial distribution were observed and justified.
\end{abstract}

Keywords: 3D EBSD, grain boundaries, misorientation, polycrystalline microstructure, statistical image analysis.

\section{INTRODUCTION}

The general aim of computational materials science is to create models of the respective structure and perform experiments - often complicated and expensive in reality - in a much less expensive way on computers. In many cases this goal is not limited by the lack of understanding of physical processes, but by the absence of a suitable geometric model of the structure. Despite the explosion of three-dimensional imaging techniques during the last decades, the majority of 3D models use simple mathematical schemes to build structures by generic procedures calibrated to the observed data only by a few parameters measured from $2 \mathrm{D}$ sections of the material. The evident errors are later corrected by manual modifications.

Destructive tomography of materials exploiting focused ion beam (FIB) installed on a scanning electron microscope (SEM) promises the possibility of full microstructure reconstruction of granular materials with all features of grains. A sufficiently dense set of 2D images of the specimen prepared via FIBslicing can be used to reconstruct the grain boundaries.
Additional measurement tools can provide information on further properties such as chemical composition or crystallographic orientation of the crystal lattice in the grains.

The electron backscattered diffraction method (EBSD) is based on a quite complex combination of inelastic scattering and channeling processes to get to orientation of a known crystal lattice from the spatial distribution of scattered electrons of primal beam. The projection of such electrons to the detector plane is called Kikuchi pattern. The method itself is even older than SEM, on whose platform it is based nowadays. Originally, the Kikuchi patterns were searched and published as a proof of the quantum mechanics, specifically de Broglie hypothesis (Nishikawa et al., 1928). Utilizing new types of detectors and mainly robust computerization, EBSD became one of the most frequent analytical techniques installed on SEM and its use covers microstructure analysis, phase analysis, textures, grain boundaries and other topics of materials science (Schwartz et al., 2009; Engler et al., 2010).

The possibility of 3D imaging was achieved by 
joining EBSD and focused ion beam tomography (FIB). The pioneering work of Zaefferer (Zaefferer, 2005) was quickly followed by other authors, nevertheless the number of publications is around ten articles a year up to now, which describes the experimental and computational demandingness of the topic. The crucial task became and still is the possibility to image 3D data and to process them. Surprisingly, not the excels producers of EBSD hardware, who are supplying software for EBSD in 2D, but independent consortia producing the software DREAM.3D got supremacy in the field (Donegan et al., 2013; Mingard et al., 2018).

Note that the benefit is the possibility of using xenon plasma FIB. Whereas conventional gallium liquid metal ion source FIB can cut volumes up to around $80 \times 80 \times 80 \mu \mathrm{m}^{3}$, the plasma sources allow to cut volumes having a width of up to $400 \mu \mathrm{m}$ with the highest acceleration voltage. There has to be emphasized that the beam of plasma FIB (P-FIB) is not only approximately fifty times stronger, but also fifty times wider. However, this does not restrict our application (Burnett et al., 2016). Being able to scan exceptionally big volumes opens new fields for study. The grains can be much larger, while still acquiring reasonable statistics in reconstructed volumes. Of course, if the grains are small, the statistics will get much better.

Tomographic imaging through FIB slicing gives the information about the microstructure and the 3D morphology of the grains. Moreover, EBSD measurements provide information about the lattice orientation within the grains and relations between the grains. All these data sets contain enough information for calculating the desired mechanical properties of the sample. Thus, the methodology described in the present paper provides a proper basic for developing suitable stochastic models of the 3D microstructures under consideration.

The material investigated in this study is the aluminum alloy $\mathrm{Al}-3 \mathrm{Mg}-0.2 \mathrm{Sc}$, which is an alloy known for its superplasticity at high deformation rates. The material was selected due to its fine-grained microstructure. The fine microstructure is important for 3D experiments as reasonable 3D-EBSD mapping on Ga-FIB is realistic in a cube with edge length around $25 \mu \mathrm{m}$. Finally, much fluent Xe-FIB was exploited, nevertheless the size of observed features remain the main issue of $3 \mathrm{D}$ experiment.

Fine-grained microstructure was prepared by a well-known severe plastic deformation method of equal channel angular pressing (ECAP) (Valiev et al., 2006), which uses a bent or even geniculate dye.
The method produces an intensive deformation in the whole volume of the sample, and, especially, the $\mathrm{B}_{\mathrm{C}}$ procedure - the sample is rotated about $90^{\circ}$ around the press axis and produces a homogeneous microstructure of equiaxed grains. Compared to the conventional technique of rolling, the ECAP method produces predominantly high-angle grain boundaries, important for superplastic behaviour, i.e., the ability to deform the material to several hundred percent.

Additives to the major element - in our case aluminum - are important for the stability of alloys. It was found out that pure aluminum can be prepared by ECAP with similarly small grains, but the microstructure is not stable even when the ECAP process is repeated (Kawasakia et al., 2009). The resistance to dynamic recovery and grain growth can be improved by adding a small amount of magnesium. This allows to obtain grain sizes below one micrometer, but the number of ECAP passes to get homogeneous microstructures increases (Xu et al., 2011). The stabilization of microstructure at temperatures below $500{ }^{\circ} \mathrm{C}$ can be achieved by adding some scandium (Lee et al., 2002). The superplastic deformation appears up to $300{ }^{\circ} \mathrm{C}$ and strain rates close to $10^{-2} s^{-1}$, which is two orders of magnitude higher than the strain rates typically seen for superplastic sliding of high-angle grain boundaries.

To test the 3D-EBSD procedure, a set of differently annealed samples was prepared. Small grains need to be distributed homogeneously in the properly annealed material for superplastic deformation. Such microstructure was obtained in the $\mathrm{Al}-3 \mathrm{Mg}-0.2 \mathrm{Sc}$ alloy by annealing $1 \mathrm{~h}$ at $400{ }^{\circ} \mathrm{C}$ (Dám et al., 2013; Horita et al., 2000). Thus, the microstructure after higher temperature annealing contains big grains, whereas the microstructure annealed at lower temperature contains smaller grains with areas containing plastic deformation from ECAP. The EBSD maps of samples annealed at $500{ }^{\circ} \mathrm{C}$ and $300{ }^{\circ} \mathrm{C}$, respectively, confirmed the general expectations.

Here we deal with a polycrystalline material, whose structuring elements are grains. The grains are created within the processing of material (solidification, recrystallization, etc.), so we expect that the grains' interiors have generally the same or very similar properties (e.g. crystallographic orientation) and well-defined boundaries. Conventional metallography observes grain boundaries after some processing and thus established properties of grain interior (for example grain size). Our data was obtained by the EBSD method and we have the set of orientations of measured points in a 3D matrix (voxels) without given commonly understood grains. The EBSD 
method constructs the grains by collecting the points of the same orientations in the frame of a given misorientation threshold and amount of measured points necessary for a single grain. Such procedure can be, of course, varied by the selected misorientation threshold or minimal grain size. A usual value to select the grains is the misorientation of $5^{\circ}$ in the EDAX OIM software, but other values can be selected with respect to the physical reality description. We understand by a grain not only the "physical" grain, but also the construct of the grain selection procedure/algorithm. Grains are created by the described procedure even in the area without any material, so it is obvious that they are influenced by measurement noise.

The grains obtained under various levels of the misorientation tolerance (i.e. with various definitions of a grain) are examined in our paper by a statistical evaluation of the microstructure including the geometry of grains and misorientations of grain boundaries. It should be mentioned that the misorientation between the measured points used as a criterion to separate the grains is a different property than the later used misorientation between the grains, which is a property of the grain boundaries.

EBSD is the most suitable and widely used experimental technique for the determination of microtexture. (Engler et al., 2010) use this notion in connection with an approach which deals with orientation statistics of a population of individual grains and encompasses the spatial location of these grains. We contribute to the methodology of a statistical evaluation of microstructure and texture in the given experiment using the DREAM3D software and methods of spatial statistics of point patterns implemented in R. Here point patterns represent the locations of individual grain boundaries. Some observed inhomogeneities in both grain orientations and spatial distribution of grain boundaries are interpreted and justified.

\section{EXPERIMENTAL}

The initial material was cast from elements (purity $99.95 \%$ ) in induction furnace under argon protective atmosphere. Ingots were cut by a electrical discharge machine to minimize both contamination by abrasive and deformation of surface layer of the material. The ECAP process was performed in our own manufactured form with $\mathrm{B}_{\mathrm{C}}$ rotation option between passes, see Fig. 1. The deformation machine INSTRON 5882 was used as a press to get stable pressing conditions. The number of passes for a homogeneous, equiaxed microstructure - namely 8 passes - was determined in previous studies (Dám et al., 2013; Horita et al., 2000).

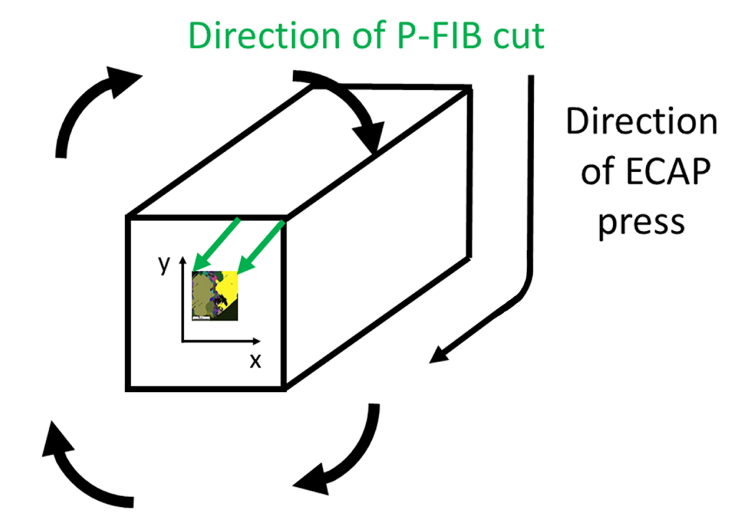

Rotations in $\mathrm{B}_{\mathrm{C}}$ ECAP process

Fig. 1. Sketch of the 3D-EBSD mapped area with respect to the position in the sample processed by the $B_{C}$ route of the ECAP process.

The reference samples for 2D EBSD were prepared by standard metallography following the procedure for aluminum alloys. Polishing was finished with colloidal silica and Kroll's reagent as this material has a strong tendency to smear and subsequent surface recrystallization. Samples for 3D-EBSD were cut by the electric discharge machine ZAP BP 05dw and the surface was polished by sand paper of grade P4000 before FIB processing.

3D-EBSD tomography was performed with the scanning electron microscope Tescan FERA 3, which is equipped with a xenon plasma FIB. The system produced by EDAX with a DigiView IV camera was used for EBSD. The maximum acceleration voltage for ions - $30 \mathrm{kV}$ - was applied to manipulate the sample. The maximum current - $2 \mu \mathrm{A}$ - was used to prepare the tomography volume and a current 300 of nA was used to slice samples. The required quality of cuts was achieved by the application of silicon masks. This method developed by Tescan prevents contact of ion beam tails with the cross-cut in sample and creation of milling artifacts, here curtains. Nevertheless, the curtaining effect is still present as it can be seen in Fig. 2 (the banked black strips). Raw data were exported (as text ang-format) to DREAM.3D software (Groeber et al., 2014) and processed there.

A pillar for 3D-EBSD was cut without tilting the stage. Such experiment geometry causes inclined tomography pillars, because the ion tube is inclined by $55^{\circ}$ toward the electron tube. The mapped area is then selected from the larger surface of the pillar sliced by FIB. 


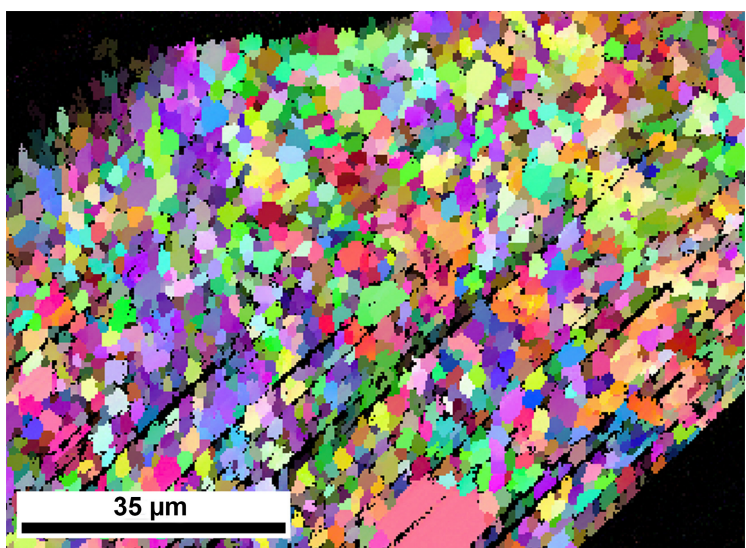

(a)

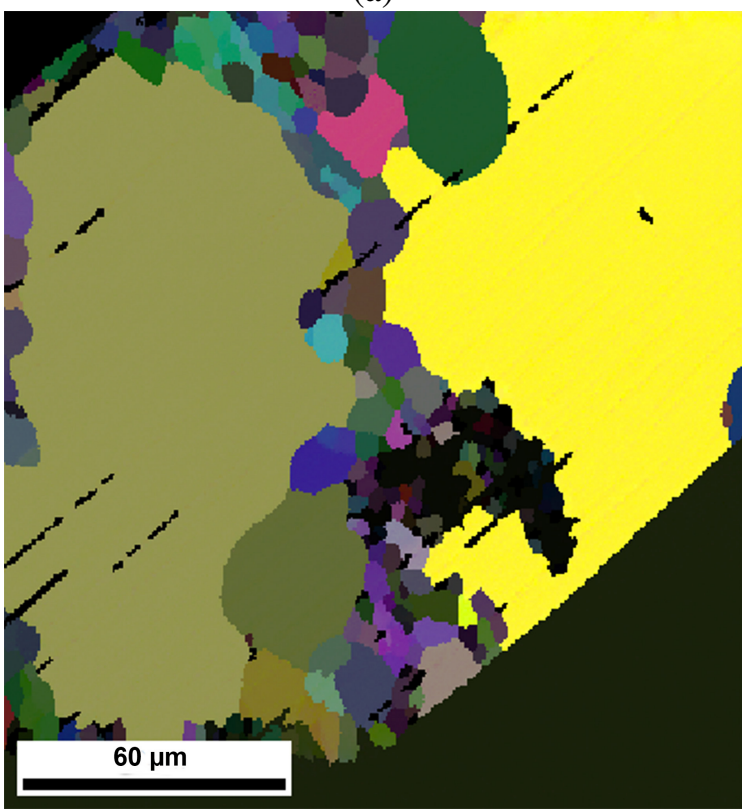

(b)

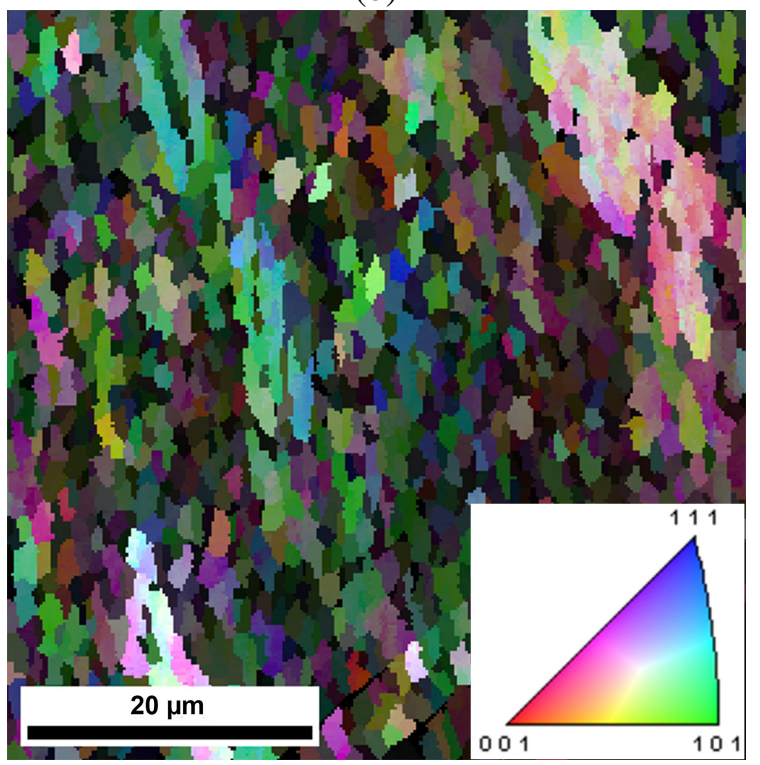

(c)

Fig. 2. Three samples of the aluminum alloy Al-3Mg$0.2 \mathrm{Sc}$. The samples have been annealed $1 \mathrm{~h}$ at $400^{\circ}$ (a), $1 \mathrm{~h}$ at $500{ }^{\circ} \mathrm{C}(\mathrm{b})$ and $1 \mathrm{~h}$ at $300^{\circ} \mathrm{C}(\mathrm{c})$. The color scale given in the inlet of Fig. 1c is valid for all parts of Fig. 2.

The sample annealed $1 \mathrm{~h}$ at $400{ }^{\circ} \mathrm{C}$ was sliced with a voxel size of $300 \mathrm{~nm}$ (i.e., the progression step in all three dimensions was $300 \mathrm{~nm}$ ). The resulting microstructure has an average grain size of $(3 \pm 2) \mu \mathrm{m}$. The size of the sliced area was $98.4 \times 71.7 \mu \mathrm{m}^{2}$ where 117 slices were mapped, i.e., the size of the specimen was $98.4 \times 71.7 \times 35.1 \mu \mathrm{m}^{3}$ (see Fig. 3 ).

The sample annealed $1 \mathrm{~h}$ at $500{ }^{\circ} \mathrm{C}$ has much less homogeneous microstructure than was expected. The size of the mapped area was $169 \times 182,5 \mu \mathrm{m}^{2}$ with $500 \mathrm{~nm}$ voxels in 134 slices. The average grain is size (70 \pm 40$) \mu \mathrm{m}$, but the vast majority of detected grains in one slice has a size below $10 \mu \mathrm{m}$. Two enormous grains lead to the large average value of grain size.

The sample annealed $1 \mathrm{~h}$ at $300{ }^{\circ} \mathrm{C}$ shows the most complicated microstructure. It was sliced on an area of approximately $52 \times 52 \mu \mathrm{m}^{2}$ with a voxel size of 200 $\mathrm{nm}$. It was necessary to lower voxel as the grain is small and a bigger step led to under-sampling. There were 255 mapped slices. The average grain size is $(1,4 \pm 0,7) \mu \mathrm{m}$ and there clusters of grains of similar orientation can be seen in inverse pole figure color coded orientation maps as are in Fig. 2.

The first attempt was decided to investigate mainly the sample annealed $1 \mathrm{~h}$ at $400{ }^{\circ} \mathrm{C}$.

\section{DATA PREPARATION}

The main information obtained from 3D-EBSD is the spatial orientation of measured points, which is represented in Euler angles (Engler et al., 2010). The 3D-EBSD method provides data in the form of 2D slices, which can be subsequently joined to a 3D image for further analyses. Unfortunately, the measured material is influenced by current and heat during FIB manipulation and subsequent EBSD mapping, which leads to some translation misalignment between adjacent 2D slices. In order to correct this misalignment and form a representative 3D image, the filter 'Align Section (Misorientation)' in DREAM.3D was used.

For the detection of individual grains, the filter ' Segment Features (Misorientation)' with misorientation tolerance of $2^{\circ}$ was used, where by misorientation of two neighboring points, the difference between the orientations of these points is meant (Šedivý et al., 2013). In the following section, the difference between the detection of grains in 
2D and 3D will be briefly discussed. The grains with volume less than 7 voxels are removed from the analysis using the filter 'Minimum Size' because of noise appearing in the data. Further, the characteristics described in the following section, namely the volume, surface area, volume-equivalent diameter, sphericity, number of neighbours and centroid of each grain as well as the misorientations of neighbouring grains are obtained by corresponding filters in DREAM.3D.

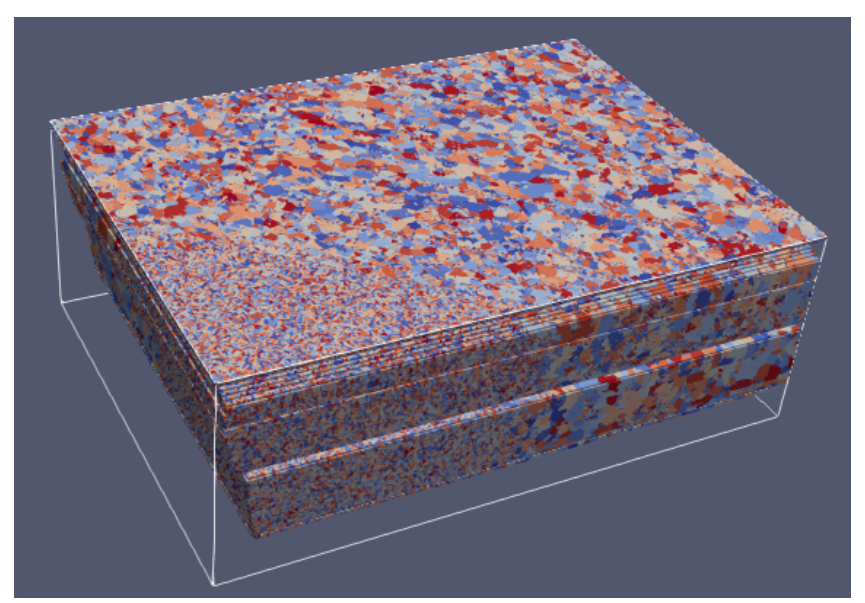

(a)

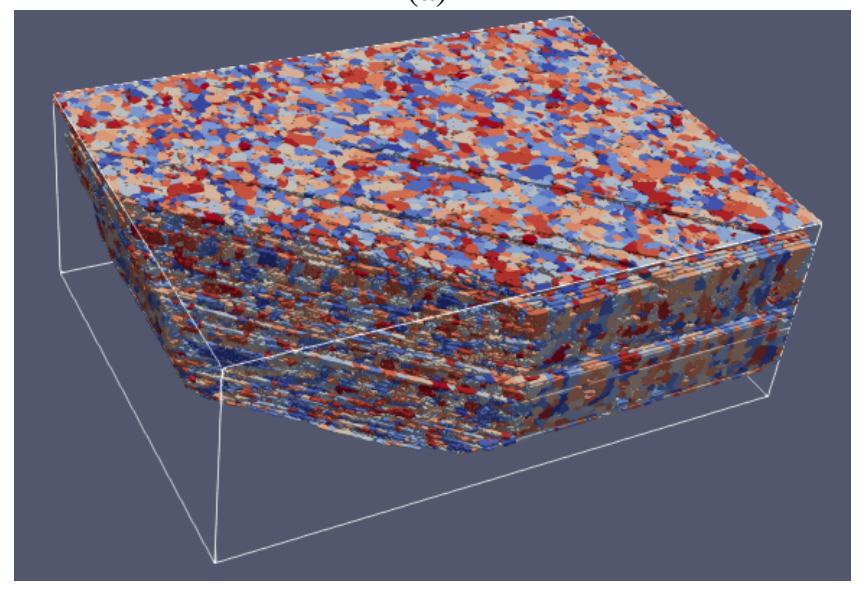

(b)

Fig. 3. The data analysed in this paper has been processed by DREAM.3D. The original data (1 593 582 grains) (a) and the data without the noise as well as without the small grains (74 579 grains) (b). Grains are coloured at random.

\section{GRAIN DETECTION IN 2D AND 3D}

In the last few decades the 2D-EBSD method has been frequently used for the microstructure analysis of crystalline materials. Nowadays, it is even possible to use the 3D-EBSD method, which allows to analyze the whole volume of a specimen. It is quite natural to compare single 2D slices created by the process using 3D-EBSD to 2D specimens obtained from 2D-EBSD.

The basic question is whether similar results of the given specimen segmentation into the grains using the same misorientation tolerance level in 2D and 3D can be achieved. There is also the question whether it is reasonable to use the same choice of misorientation level in 2D and 3D in order to compare these two methods. The answers surely depend on the choice of the investigated material.

To compare the results obtained in $2 \mathrm{D}$ and $3 \mathrm{D}$, the process the whole 3D specimen in DREAM.3D by using the filters described in the previous section. Then an only one single slice from the processed 3D data is cut out. This slice is then processed separately, i.e., we only consider the voxels from the slice for the segmentation into grains, using the same filters and the same settings as in 3D. In the following, the results obtained by these two processing methods are compared to each other.

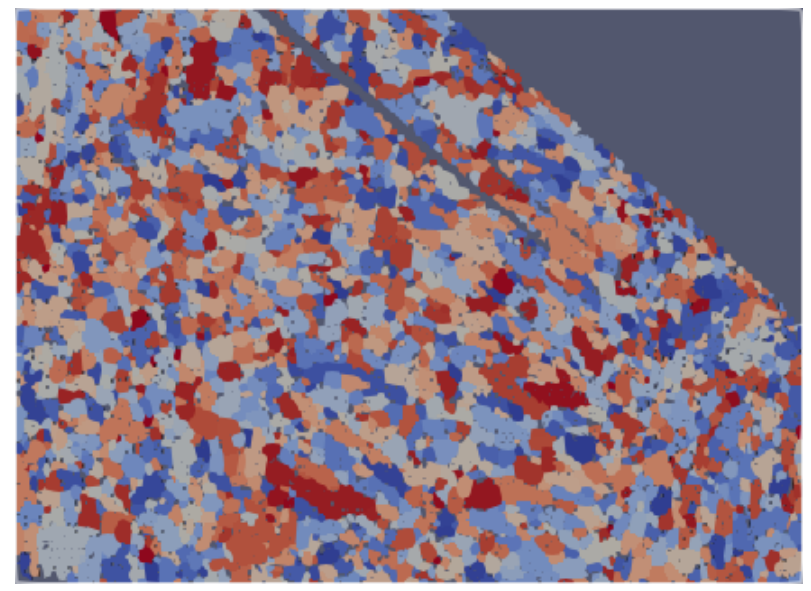

(a)

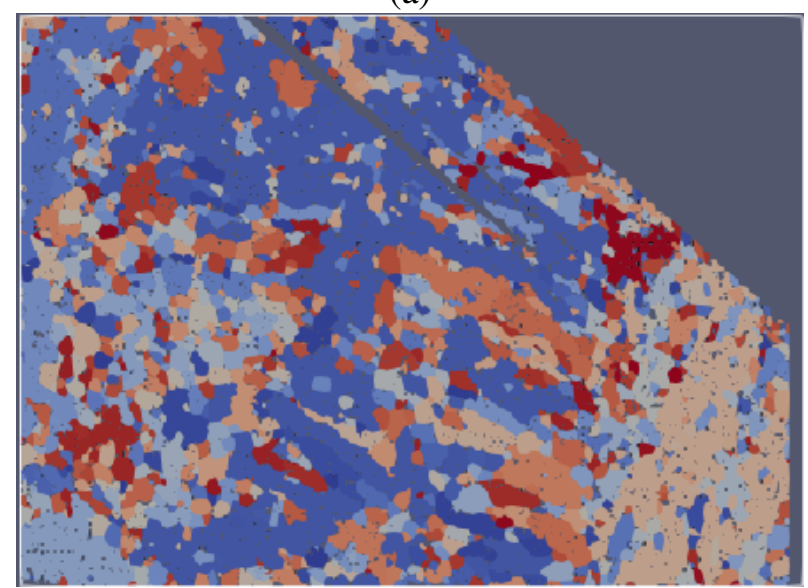

(b) 
Fig. 4. Segmentation of a sampled slice (namely slice No. 102) with the misorientation tolerance equal to $5^{\circ}$. Segmentation in 2D (2149 grains) (a) and segmentation in $3 D$ (1412 grains) (b).

As seen in Fig. 4, the choice of the (classical) misorientation level equal to $5^{\circ}$ for both the $2 \mathrm{D}$ and 3D methods does not lead to similar results. In the three-dimensional case, a microstructure with significantly larger grains than in the two-dimensional segmentation is obtained. That brings the idea of studying the number of grains identified by the $2 \mathrm{D}$ and 3D segmentations under different conditions.

For further analysis, the slices No. 2, 12, 22, 32, $\ldots, 102$ and 112 are considered and their properties are studied under different misorientation levels, namely $1^{\circ}, 1.5^{\circ}, 2^{\circ}, 3^{\circ}, 4^{\circ}, 5^{\circ}, 6^{\circ}, 8^{\circ}$ and $10^{\circ}$.

Before starting to compare the number of grains, it is necessary to state which grains are important for the analysis. As mentioned above, the specimen contain a lot of noise (see Fig. 2), whose presence is negligible but can distort the results. Therefore the following corrections have been made: First, only the grains which are not too small, more precisely the grains consisting of at least 7 voxels in 3D processing, were taken into account. An analogous condition was used in the 2D case, where only the grains consisting of at least 4 voxels were considered. Note that after deleting the small grains, DREAM.3D makes a new "cleaned" slice which is further analyzed. However, since there is no rule how to set the relation between the minimal numbers of voxels in 2D and 3D, and since the number of grains, obviously, strongly depends on the choice of the minimal numbers mentioned above, the analysis cannot be built only on this approach. Therefore, a second correction was applied as follows. The grains are ordered from the largest to the smallest ones, and then the number of grains was calculated starting with the largest ones and going grain by grain to the smaller ones until their total area covers $90 \%$ of the whole slice. In this way, the impact of noise is eliminated.

Using the latter correction, we plot the dependence of the mean number of grains in single 2D slices on the misorientation tolerance level for both 2D and 3D segmentations (see Fig. 5). It can be seen that the difference between the methods is significant. While the 3D segmentation identifies approximately 2000 grains for the misorientation level $1^{\circ}$, the $2 \mathrm{D}$ segmentation produces almost 500 grains more. In order to obtain 2000 grains by the 2D segmentation, the misorientation level of $2^{\circ}$ has to be considered. While the 3D segmentation identifies 1500 grains for the misorientation level of $2^{\circ}$, the $2 \mathrm{D}$ segmentation requires the misorientation level of $4^{\circ}$ to obtain the same number of grains. Such behaviour can be observed in the whole plot in Fig. 5. From the presented simulation study can be concluded that in order to identify the same number of grains by the 2D and 3D methods in the material specimen analyzed in this paper, the misorientation level considered for the 2D segmentation should be approximately twice as large as in the case of the 3D segmentation.

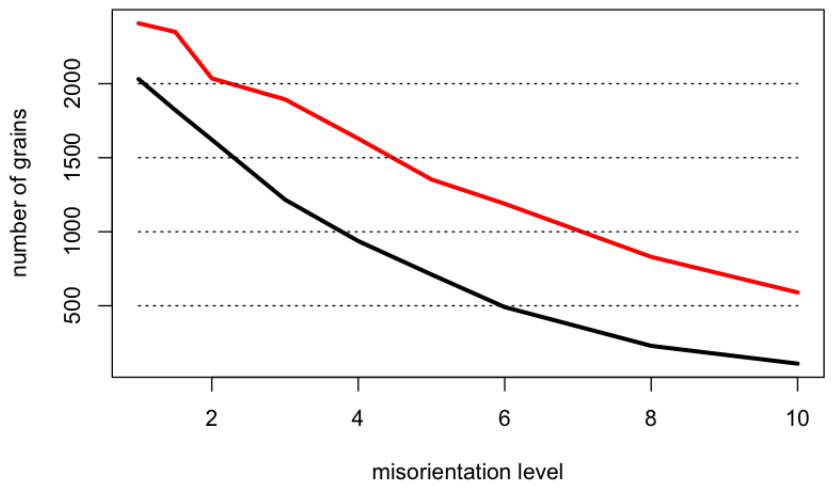

Fig. 5. Dependence of the mean number of grains in single $2 D$ slices on the misorientation tolerance level while processing by the $2 D$ method (red line) and the $3 D$ method (black line). Note that we only consider the largest grains which cover in total $90 \%$ of the whole slice.

\section{STATISTICAL ANALYSIS OF THE MICROSTRUCTURE}

The results of a quantitative microstructure analysis of the sample annealed for $1 \mathrm{~h}$ at $400{ }^{\circ} \mathrm{C}$ is presented in this section. The DREAM.3D software for descriptive statistics and the $R$ package spat stat for a more sophisticated analysis of spatial statistics is being used here. The analyses can be roughly divided into two parts, one mostly concerning characteristics of individual grains, and the other one based on grain faces (parts of grain boundaries).

\section{INDIVIDUAL GRAIN CHARACTERISTICS}

The specimen shown in Fig. 3b, has 74579 grains, where this sample is denoted by G400. The following two tables, Tab. 1 and 2, show results for some basic geometrical characteristics, denoted as: "eqd" equivalent diameter, "non" number of neighbours, "sph" sphericity, "svr" surface to volume ratio, "sur" surface area, "vol" volume. Edge effects are not corrected since a very large number of grains is measured. In Tab. 1 some statistics of grains from the whole sample G400 are presented. The specimen 
contains 127 small grains, which are included in a bigger grain. Therefore these grains have only one neighbour.

\begin{tabular}{|c|c|c|c|}
\hline & $\begin{array}{l}\text { eqd } \\
{[\mu m]}\end{array}$ & non & \\
\hline Min & 0.71 & 0.1 & \\
\hline Max & 13.22 & 852 & \\
\hline Median & 1.16 & 10 & \\
\hline Mean & 1.33 & $11.81 \quad 0.5$ & \\
\hline \multirow[t]{2}{*}{ SD } & 0.62 & $10.19 \quad 0.1$ & \\
\hline & $\begin{array}{l}\mathrm{svr} \\
{\left[\mu m^{-1}\right]}\end{array}$ & $\begin{array}{l}\text { sur } \\
{\left[\mu m^{2}\right]}\end{array}$ & $\begin{array}{l}\text { vol } \\
{\left[\mu m^{3}\right]}\end{array}$ \\
\hline Min & 1.85 & 0.09 & 0.19 \\
\hline Max & 26.67 & 3072.24 & 1210.65 \\
\hline Median & 9.88 & 6.84 & 0.81 \\
\hline Mean & 10.07 & 12.49 & 2.34 \\
\hline SD & 3.40 & 25.97 & 8.71 \\
\hline
\end{tabular}

Table 1. Minimum, maximum, median, mean and standard deviation (SD) of basic geometrical characteristics of grains from the sample G400.

The total volume of all segmented grains is equal to $174111.84 \mu^{3}$. The majority of grains is very small in the sense that 27799 grains fill up less than $5 \%$ of the overall volume. On the other hand, 16891 grains form three quarters of the overall volume, where we denote this part of the sample by G400-3/4. In Fig. 6 histograms are given for four geometrical characteristics of grains for both the samples G400 and G400-3/4.

The values of some basic characteristics of the biggest grains (with volume $>200 \mu \mathrm{m}^{3}$ ) are given in Tab 2.

\begin{tabular}{rrrrrr}
$\begin{array}{r}\text { vol } \\
{\left[\mu \mathrm{m}^{3}\right]}\end{array}$ & non & sph & $\begin{array}{r}\text { sur } \\
{\left[\mu \mathrm{m}^{2}\right]}\end{array}$ & $\begin{array}{r}\text { eqd } \\
{[\mu \mathrm{m}]}\end{array}$ & $\begin{array}{r}\text { svr } \\
{\left[\mu m^{-1}\right]}\end{array}$ \\
\hline 1210.65 & 852 & 0.16 & 3072.24 & 13.22 & 2.89 \\
849.34 & 680 & 0.17 & 2246.22 & 11.75 & 3.03 \\
665.87 & 649 & 0.16 & 1986.39 & 10.83 & 3.54 \\
439.37 & 314 & 0.20 & 1177.11 & 9.43 & 3.13 \\
380.92 & 289 & 0.21 & 987.66 & 8.99 & 3.15 \\
351.78 & 260 & 0.22 & 838.35 & 8.76 & 3.09 \\
341.66 & 302 & 0.20 & 1030.95 & 8.67 & 3.49 \\
329.64 & 480 & 0.20 & 1085.04 & 8.57 & 3.46 \\
240.17 & 239 & 0.18 & 739.35 & 7.71 & 4.34 \\
236.60 & 268 & 0.22 & 738.72 & 7.67 & 3.61 \\
213.79 & 155 & 0.31 & 499.59 & 7.42 & 2.60 \\
202.99 & 134 & 0.29 & 558.36 & 7.29 & 2.89 \\
\hline
\end{tabular}

Table 2. Values of basic geometrical characteristics for twelve biggest grains from sample G400.

Tab. 3 depicts correlations between basic grain characteristics.

\begin{tabular}{ccccccc} 
& 1 & 2 & 3 & 4 & 5 & 6 \\
\hline corr & 0.97 & -0.21 & 0.84 & -0.30 & -0.39 & 0.91 \\
\hline
\end{tabular}

Table 3. Pearson correlation coefficients between basic geometrical characteristics of grains from sample G400. The notation is as follows: correlation between volume and surface area (1), volume and sphericity (2), volume and number of neighbours (3), sphericity and surface area (4), sphericity and number of neighbours (5), surface area and number of neighbours (6).

Note that the sphericity is defined by the formula

$$
\Phi=\frac{\pi^{1 / 3}(6 V)^{2 / 3}}{A}
$$

where $\mathrm{V}$ and $\mathrm{A}$ is the volume and surface of the considered grain, respectively. Thus, theoretically, it holds $\Phi \leq 1$. However in our data set, we have 24 grains with sphericity larger than one. It is probably caused by the voxel-based calculation of volume and surface. The values were obtained by DREAM.3D which uses filters with edge corrections. Since all the 24 grains with $\Phi>1$ are very small and located at the boundary of the specimen, the calculation of their characteristics may be biased.
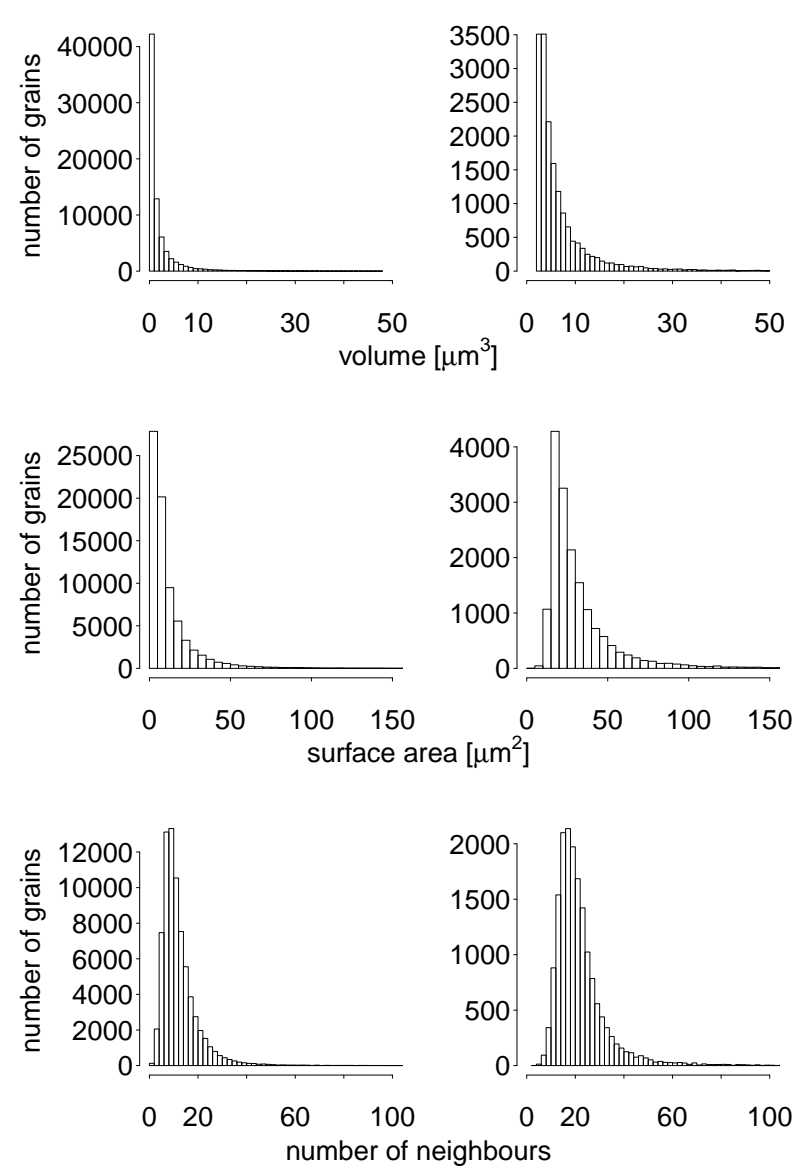


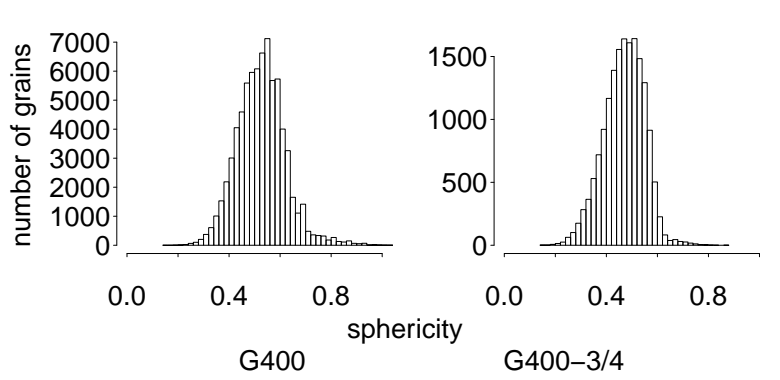

Fig. 6. Histograms of grain statistics. For better visualisation, there are 158, 200 and 28 grains omitted in the histograms of volume, surface area and number of neighbours, respectively, because these grains are outliers with very large corresponding statistics.

\section{CHARACTERISTICS OF INDIVIDUAL GRAIN FACES}

For each pair of neighbouring grains the common face (a part of the network of grain boundaries) is considered. The location of each face is given by a representative point with coordinates

$\left[x_{1}+\frac{e d_{1}\left(x_{2}-x_{1}\right)}{e d_{1}+e d_{2}}, y_{1}+\frac{e d_{1}\left(y_{2}-y_{1}\right)}{e d_{1}+e d_{2}}, z_{1}+\frac{e d_{1}\left(z_{2}-z_{1}\right)}{e d_{1}+e d_{2}}\right]$,

where $x_{i}, y_{i}, z_{i}$ are the centroid coordinates and $e d_{i}$ is the volume-equivalent diameter of the $i$-th grain in the pair, $i=1,2$.

For a face we define the volume neighbour ratio (VNR) by

$$
V N R=\left(\frac{\max \left\{\left|C_{1}\right|,\left|C_{2}\right|\right\}}{\min \left\{\left|C_{1}\right|,\left|C_{2}\right|\right\}}-1\right)^{1 / 2},
$$

where $C_{1}, C_{2}$ are two neighbouring grains creating a common face, $|C|$ is the volume of grain $C$.

Tab. 4 describes statistics of textural and geometrical characteristics from a sample of 440506 faces (denoted as F400) identified on specimen G400. Note that edge effects are not corrected. The misorientation was obtained by DREAM.3D using the filter 'Find Feature Neighbor Misorientations'. The misorientations of two features/grains are evaluated from the average orientation of the grains, therefore the derived misorientation could be lower than the chosen misorientation treshold. In our dataset 2786 faces have misorientation lower than $2^{\circ}$. In most cases the faces have small areas. Only one face has a misorientation of $0^{\circ}$.

\begin{tabular}{llll} 
& $\begin{array}{l}\text { misor } \\
{\left[{ }^{\circ}\right]}\end{array}$ & $\begin{array}{l}\text { area } \\
{\left[\mu \mathrm{m}^{2}\right]}\end{array}$ & VNR \\
\hline Min & 0 & 0.09 & 0 \\
Max & 62.56 & 107.82 & 80.03 \\
Median & 23.99 & 0.63 & 1.65 \\
Mean & 29.63 & 1.06 & 2.59 \\
SD & 20.25 & 1.52 & 3.67
\end{tabular}

Table 4. Minimum, maximum, median, mean and standard deviation $(S D)$ of textural (misorientation, briefly called 'misor') and geometrical (area, VNR) characteristics of faces from sample F400.

The total area of all faces in sample F400 is equal to $465786.54 \mu \mathrm{m}^{2}$. Altogether, 133320 faces contribute to this total area by less than $5 \%$ and 150713 faces create three quarters of the total area (sample denoted as F400-3/4). Among these 150713 faces the smallest one has an area of $0.99 \mu^{2}$.

Fig. 7 shows kernel density estimators of misorientation, area and VNR for both samples F400 and $\mathrm{F} 400-3 / 4$

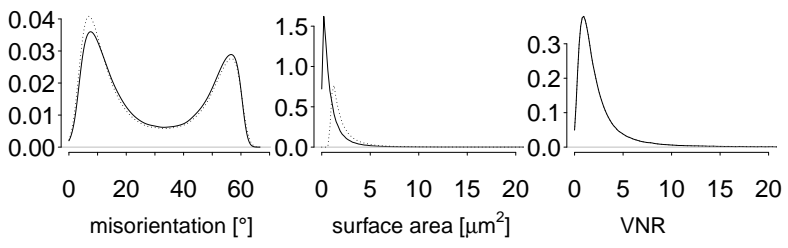

Fig. 7. Kernel density estimators of face statistics for sample F400 (solid line) and sample F400-3/4 (dotted line).

According to their misorientation, the faces are split into three classes, where the groups $10^{\circ} \pm 5^{\circ}$ (LAGB) and $55^{\circ} \pm 5^{\circ}$ (HAGB) will be considered and the rest, see Tab. 5. Notice that the abbreviation "HAGB" refers to a particular selection of misorientation contrary to the usual definition of high-angle grain boundary (Engler et al., 2010).

\begin{tabular}{cc} 
& number of faces \\
\hline total & 440506 \\
LAGB & 138333 \\
HAGB & 115219 \\
the rest & 186954 \\
\hline
\end{tabular}

Table 5. Decomposition of faces from sample F400 into three classes.

In Tab. 6 the results obtained for the correlation between different face characteristics in sample F400, separately for the classes LAGB and HAGB are stated. It is concluded that the Pearson correlation coefficiens in both classes do not differ dramatically. 


\begin{tabular}{lllllll} 
& A & B & C & D & E \\
\hline LAGB & -0.07 & -0.01 & -0.01 & -0.03 & -0.01 \\
HAGB & 0.03 & 0.02 & 0.02 & 0.02 & 0.03 \\
\hline & & & & & & \\
& $\mathrm{F}$ & $\mathrm{G}$ & $\mathrm{H}$ & $\mathrm{I}$ & $\mathrm{J}$ & $\mathrm{K}$ \\
\hline LAGB & 0.19 & 0.20 & 0.02 & 0.20 & 0.79 & 0.39 \\
HAGB & 0.18 & 0.17 & 0.01 & 0.18 & 0.78 & 0.38 \\
\hline
\end{tabular}

\begin{tabular}{lllll} 
& $\mathrm{L}$ & $\mathrm{M}$ & $\mathrm{N}$ & $\mathrm{O}$ \\
\hline LAGB & 0.97 & 0.58 & 0.86 & 0.48 \\
HAGB & 0.97 & 0.54 & 0.85 & 0.46 \\
\hline
\end{tabular}

Table 6. Pearson correlation coefficients between characteristics of grain faces from sample F400. The notation is as follows: Pearson correlation coefficient between misorientation and face area $(A)$ misorientation and volume difference (B), misorientation and difference of volume-equivalent diameters $(C)$, misorientation and sphericity difference $(D)$, misorientation and difference in number of neighbours (E), face area and volume difference $(F)$, face area and difference of volume-equivalent diameters $(G)$, face area and sphericity difference $(H)$, face area and difference in number of neighbours (I), volume difference and difference of volumeequivalent diameters $(J)$, volume difference and sphericity difference $(K)$, volume difference and difference in number of neighbours $(L)$, difference of volume-equivalent diameters and sphericity difference $(M)$, difference of volume-equivalent diameters and difference in number of neighbours $(N)$, sphericity difference and difference in number of neighbours $(O)$.

Let PF400 denote the point pattern of representative points of sample F400 and divide this pattern into subpatterns PF400L, PF400H and PF400R according to the misorientation classes LAGB, HAGB and the rest, respectively. The $\mathrm{R}$ package 'Spatstat' can be used for the estimation of various statistics of 3D point processes, cf. Chiu et al. (2013). Histograms of nearest neighbour distance are plotted in Fig. 8 for the subpatterns of representative points of faces PF400. The empty-space function, nearest neighbour distribution function and pair correlation function are plotted in Fig. 9 for the same subpatterns.

It was observed that there is almost no difference in these characteristics between classes LAGB and HAGB. On the other hand, in Fig. 9, there is a tendency to non-regularity since the empty space function is shifted to the right in comparison to the Poisson case. This fact is caused by the presence of large grains in the specimen.

\section{DISCUSSION}

All the prepared samples from the Al-3Mg-0.2Sc alloy were successfully mapped. There was expected a locally inhomogeneous microstructure with big grains (annealing at $500{ }^{\circ} \mathrm{C} / 1 \mathrm{~h}$ ), a rather homogeneous microstructure without texture (annealing at $400{ }^{\circ} \mathrm{C}$ / $1 \mathrm{~h}$ ) and again an inhomogeneous microstructure with a significant amount of deformation stored in grains (annealing at $300{ }^{\circ} \mathrm{C} / 1 \mathrm{~h}$ ). The general expectations were satisfied. The sample annealed at $500{ }^{\circ} \mathrm{C}$ contains two big grains and despite the large observed volume we documented just a few tens of grains between them.

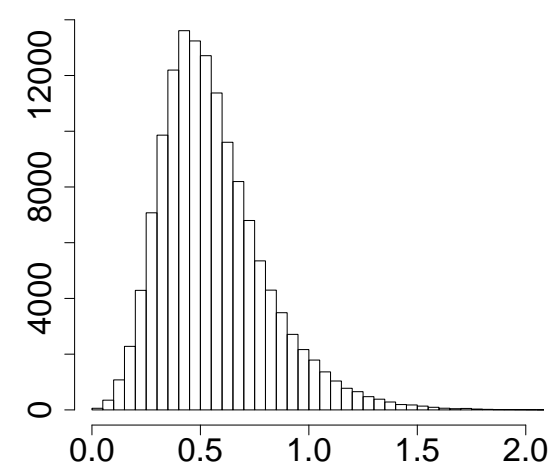

(a)

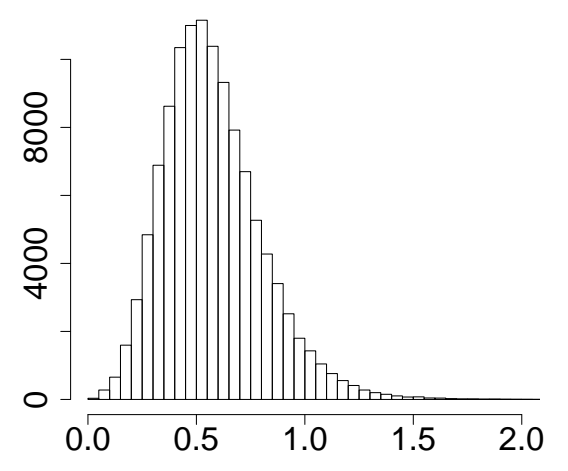

(b) 


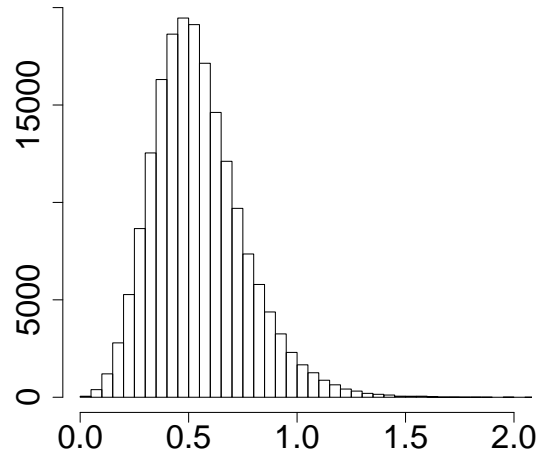

(c)

Fig. 8. Histograms of the nearest neighbour distance in the subpatterns PF40OL (a), PF400H (b), PF400R (c).

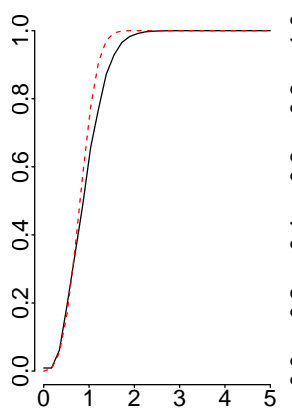

(a)

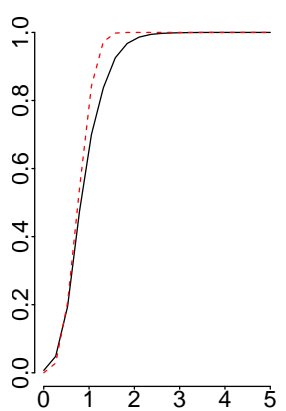

(a)

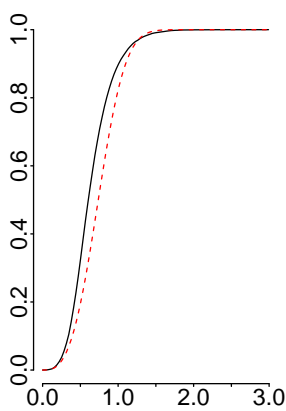

(b)

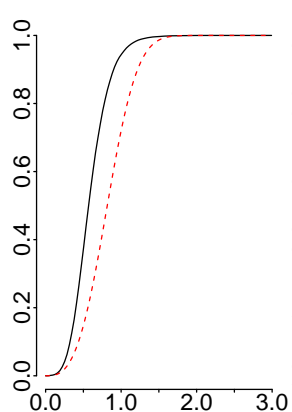

(b)

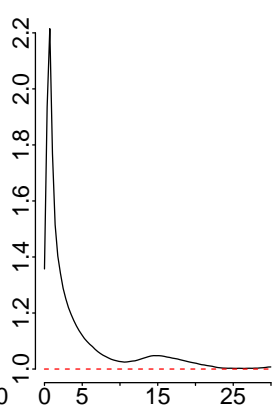

(c)

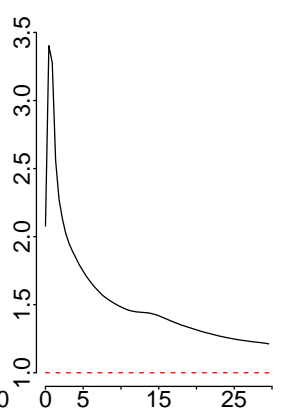

(c)

Fig. 9. The black lines represent the estimated empty space function (a), the nearest neighbour distribution function (b) and the pair correlation function (c) for the patterns PF40OL (upper row) and PF4OOH (lower row). The red cut line corresponds to the theoretical analogons of these functions for the homogeneous Poisson process with the same intensity (complete spatial randomness).

The results obtained for the sample annealed at $400{ }^{\circ} \mathrm{C}$ were more interesting. An interesting part

presents a comparison of grain segmentation in 3D and 2D. There is a higher probability to find bigger variation of adjacent points in 3D evaluation, when measured voxels have more nearest neighbours than in $2 \mathrm{D}$. Thus the obtained results are not completely surprising, mainly if the noise and experimental error of particular measurement are taken into account. The difference between both results is so high that the effect of misorientation thresholding will be further investigated.

The basic grain statistics showed the expected distribution of isotropic grains in the sample annealed at $400{ }^{\circ} \mathrm{C}$. There is a big amount of very small grains, which can be related to a lack of data processing (broken noisy grains), rather than simply to noise, as the properties of samples G400 and G400-3/4 are similar, see Fig. 6. The 2D analysis of grain faces showed that the microstructure is not fully homogeneous, see Fig. 7. The distribution of misorientations in cubic crystals have been investigated. In a homogeneous microstructure, a maximum at $45^{\circ}$ is expected (Mackenzie , 1958; Skrytnyy et al., 2018). Two different maxima of the misorientation distribution of grains were found. The maximum at $10^{\circ} \pm 5^{\circ}$ (LAGB) can be attributed to the incomplete destruction of the cast microstructure of the original material. Conventional metallography showed that the cast material has prolonged grain couples of a few millimetres length. Such grains decompose during the ECAP process and the new parts misorient, but the process of microstructure homogenization is not finished even after 8 ECAP passes. The maximum of misorientation at $55^{\circ} \pm 5^{\circ}$ (HAGB) corresponds to the highest misorientation between adjacent grains. It is close to the misorientation of $60^{\circ}$ of the special CSL $\Sigma 3$ boundary, but the deviation from the real $\Sigma 3$ boundary is significant and just a negligible amount of boundaries can be marked as $\Sigma 3$ boundary. This finding supports the experience of metallurgy, as we do not expect a massive amount of special boundaries, but a big amount of general high-angle grain boundaries (Dám et al., 2013; Horita et al., 2000).

As seen in Fig. 8, the behaviour of grain boundaries in all three groups is similar in the sense that the distributions of the nearest neighbour distance in the 3D space are the same for all types of grain boundaries. Fig. 9a shows that the estimate of the empty space function is mostly below the corresponding curve for the Poisson process (i.e. the model for complete spatial randomness), since the presence of a few large grains with many small faces enables the contact distance to exceed some level (between 1-5 $\mu \mathrm{m}$ ) with probability higher than in the case of the Poisson process. Similar arguments justify the differences between the curves in Fig. 9b and 9c. 
The last sample annealed at $300{ }^{\circ} \mathrm{C} / 1 \mathrm{~h}$ was found to be too noisy for the processing applied to the previous sample $\left(400{ }^{\circ} \mathrm{C}\right)$. Nevertheless, the microstructure is less homogeneous, grains are prolonged in the direction of the ECAP process as expected (Horita et al., 2000).

\section{CONCLUSIONS}

The superplastic aluminum alloy $\mathrm{Al}-3 \mathrm{Mg}-0.2 \mathrm{Sc}$ processed by ECAP and annealed was investigated by 3D-EBSD exploiting abilities of Xe-FIB. Three different microstructures were mapped successfully and the sample annealed at $400{ }^{\circ} \mathrm{C} / 1 \mathrm{~h}$ was further investigated to get a microstructure description. It was observed that to the identification of the same number of grains in the material specimen, the misorientation level needed for the conventional segmentation of $2 \mathrm{D}$ cuts is approximately twice as large as in the case of the 3D segmentation. Although the microstructure after the applied processing is expected to be homogeneous, there were found peaks in the distribution of misorientation between neighbouring grains due to the residuals of original rough cast microstructure. From the same reasons, an inhomogeneity in the size and spatial distribution of grains and grain boundaries were observed and described.

\section{ACKNOWLEDGEMENT}

The authors acknowledge the financial support for this work provided by the Czech Science Foundation, project 17-00393J. This work was supported in part by the MEYS SAFMAT CZ.02.1.01/0.0/0.0/16_013/0001406, LO1409 and LM2015088 projects (SEM maintenance).

\section{REFERENCES}

Burnett TL, Kelley R, Winiarski B, Contreras L, Daly M, Gholinia A, Burke MG, Wither PJ (2016). Large volume serial section tomography by $\mathrm{Xe}$ Plasma FIB dual beam microscopy. Ultramicrosc 161:119-29.

Chiu SN, Stoyan D, Kendall WS, Mecke J (2013). Stochastic Geometry and Its Applications, 3rd edition. Chichester: John Wiley \& Sons.

Dám K, Lejček P, Michalcová A (2013). In situ TEM investigation of microstructural behavior of superplastic Al-Mg-Sc alloy. Mat Char 76:69-75.

Donegan SP, Tucker JC, Rollett AD, Barmak K, Groeber M (2013). Extreme value analysis of tail departure from log-normality in experimental and simulated grain size distributions. Acta Mater, 61(15):5595-604.
Engler O, Randle V (2010). Introduction to Texture Analysis Macrotexture, Microtexture, and Orientation Mapping, 2nd Edition. Boca Raton: CRC Press.

Groeber MA, Jackson MA (2014). DREAM.3D: A digital representation environment for the analysis of microstructure in 3D. Integ Mat Manuf Innov 3:5-17.

Horita Z, Furukawa M, Nemoto M, Barnes AJ, Landgon TG (2000). Superplastic forming at high strain rates after severe plastic deformation. Acta Mat 48(14):3633-40.

Kawasaki M, Horita Z, Langdon TG (2009). Microstructural evolution in high purity aluminum processed by ECAP. Mat Sci Eng A 524:143-50.

Lee S, Utsunomiya A, Akamatsu $H$, Neishi K, Fukurawa M, Horita Z, Langdon TG (2002). Influence of scandium and zirconium on grain stability and superplastic ductilities in ultrafinegrained Al-Mg alloys. Acta Mat 50(3):553-64.

Mackenzie JK (1958). Second paper on statistics associated with the random disorientation of cubes. Biometrika 45:229-240.

Mingard KP, Steward M, Gee MG, Vespucci S, TragerCowan C (2018). Practical application of direct electron detectors to EBSD mapping in 2D and 3D. Ultramicrosc 184:184-242.

Nishikawa S, Kikuchi S (1928). Diffraction of cathode rays by calcite. Nature 122:726-726.

Schwartz AJ, Kumar M, Adams BL, Field DP (2009). Electron Backscatter Diffraction in Materials Science, 2nd Edition. Boston: Springer.

Skrytnyy VI, Gavrilov MV, Khramtsova TP, Kolyanova AS, Krasnov A, Porechniy SV, Yaltsev VN, (2017). Misorientation distribution function of crystals. In: 15th International SchoolConference on new materials of innovative energy: development, characterization methods and application, KnE Materials Science, 342-357.

Šedivý O, Beneš V, Ponížil $\mathrm{P}$, Král P, Sklenička V (2013). Quantitative characterization of microstructure of pure copper precessed by ECAP. Image Anal Stereol 32:65-75.

Valiev RZ, Langdon TG (2006). Principles of equalchannel angular pressing as a processing tool for grain refinement. Prog Mater Sci 51(7):881-981.

Xu Ch, Horita Z, Langdon TG (2011). Microstructural evolution in an aluminum solid solution alloy processed by ECAP. Mat Sci Eng A, 528:6059-65.

Zaefferer S (2005). Application of orientation microscopy in SEM and TEM for the study of texture formation during recrystallisation processes. Textures of matherials - ICOTOM 14. Mat Sci Forum 495-497:3-12. 